\title{
Infektion in Unfallchirurgie und Orthopädie - Technisches Management
}

\author{
Atesch Ateschrang, Christian Bahrs, Ingo Flesch, Kuno Weise
}

\section{Zusammenfassung}

Zur Therapie der Infektkomplikation in Unfallchirurgie und Orthopädie bedarf es in der Regel eines Stufenkonzeptes mit mehrfachen operativen Eingriffen, für welche eine Reihe unterschiedlicher diagnostischer Maßnahmen und Behandlungstechniken zur Verfügung steht. Deren sinnvoller Einsatz in korrekter Ausführung ist Voraussetzung für eine erfolgreiche Therapie. Die beschriebenen Verfahren zur Sanierung der Infektkomplikation, welche sämtlich ihren eigenen Stellenwert besitzen, können im Rahmen des Gesamtkonzeptes lediglich als adjuvante Möglichkeiten betrach- tet werden, die sich den chirurgischen Maßnahmen unterzuordnen haben. Dennoch sind sie aus der Palette der therapeutischen Optionen beim Infekt nicht mehr wegzudenken, was speziell für die vergleichsweise neueren Techniken wie die Jet-Lavage und die Vakuumversiegelungstechnik gilt. Unverändert haben althergebrachte Bestandteile der Therapie von Infektkomplikationen wie lokale Antibiotikaträger, Dauerdrainagen bei liegendem Metall und die offene Wundbehandlung ihren Platz. Zur korrekten Probeentnahme für die mikrobiologische Diagnostik existieren Vorgaben, an deren Inhalte erinnert werden soll.

\section{Einleitung}

Die Behandlung der postoperativen und/ oder posttraumatischen Infektkomplikation zählt auch heute noch zu den großen Herausforderungen in Unfallchirurgie und Orthopädie. Dies gilt in besonderem Maße für akute und chronische Infektionen bei komplexen knöchernen und Gelenkverletzungen sowie bei einliegenden Dauerimplantaten, speziell in der Endoprothetik. Eine eigene Problematik ist der beträchtlichen Zahl von Patienten mit multiresistenter Keimentwicklung zu eigen. Ungeachtet klar definierter Behandlungsstrategien für die chirurgische Therapie der akuten und chronischen Infektkomplikation von der notfallmäßigen Revision mit kompromissloser Nekrektomie und Sequestrektomie bis zu einem mehrschrittigen Stufenplan für die Sanierung allfälliger Knochen- und Weichteil-

OP-JOURNAL 2005; 21: 250-259

(c) Georg Thieme Verlag KG Stuttgart · New York

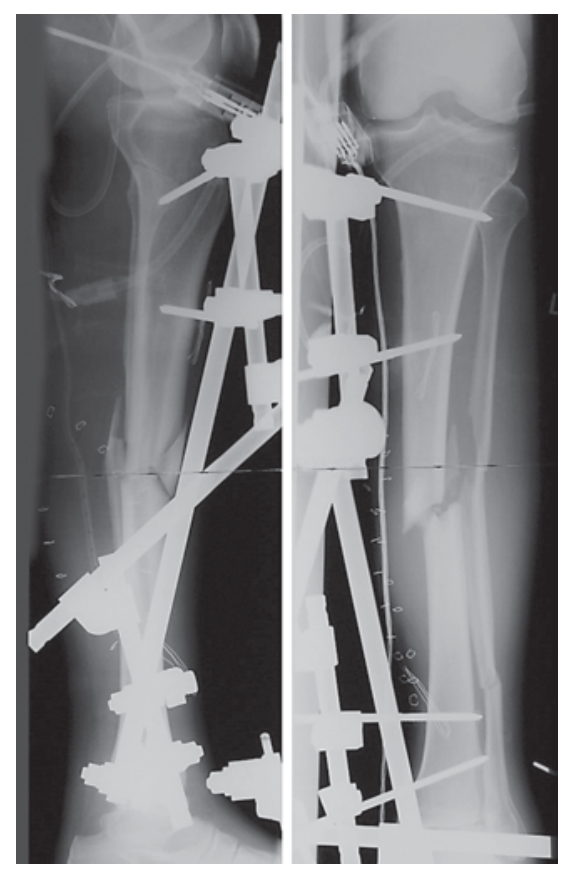

Abb.1 Röntgenaufnahme einer $\|^{\circ}$ offenen Unterschenkelfraktur links bei liegendem Fixateur. defekte sind alle notwendigen Behandlungsmaßnahmen an der jeweils vorliegenden individuellen Situation auszurichten. Aus diesen Gründen sollen die diversen Möglichkeiten verschiedener adjuvanter Techniken dargestellt und ihre jeweilige Indikation aufgezeigt werden.

Die nachfolgend beschriebenen diagnostischen Schritte und therapeutischen Optionen sind nur dann erfolgreich, wenn sie korrekt ausgeführt und in geeigneter Indikationsstellung eingesetzt werden. Dies führt zum zunächst banal erscheinenden Hinweis einer korrekten und für die Keimbestimmung optimal geeigneten Vorgehensweise für die Entnahme von Abstrichen und Gewebeproben. Hierzu existieren mittlerweile klare Vorgaben der Mikrobiologen. Weitere Empfehlungen erstrecken sich auf den korrekten Einsatz und die Darstellung der Vorzüge und Nachteile wie auch der Gefahren bei Anwendung der Jet-Lavage bis hin zu Empfehlungen für das bei dieser mechanischen Wundreinigung geeignete Medium. Zu klären ist, ob es für lokal einzulegende Antibiotikaträger heutzutage noch Einsatzmöglichkeiten gibt bzw. wie deren Indikation zu definieren oder einzugrenzen ist.

Der nach einer gründlichen Revision sowohl bei akuter Infektion als auch bei chronischer Osteitis häufig verbleibende Weichteildefekt, der entweder flächenhaft oder von einer gewissen Tiefe gekennzeichnet ist bedarf, da meist nicht unmittelbar und definitiv zu verschließen, einer temporären Wundbedeckung mit Kunsthaut oder der Ausfüllung durch eine schwammartige Folie mit inkorporierter Drainage (Vakuumversiegelungstechnik, VVS), wobei die Wunde nach Einlage des Materials mittels Operationsfolie verschlossen wird. Der Sog der Drainage führt zur Ausbildung eines Vakuums, was wiederum neben der kontrollierten Ableitung des Sekretes die Neubildung von Granulationsgewebe zur Folge hat. Gerade dieses Verfahren der VVS 
hat sich im therapeutischen Stufenplan bei der Sanierung von Infektkomplikationen einen festen Platz erobert.

Schließlich ist die Anwendung von Drainagen im Rahmen von Revisionen speziell akuter Infekte ein althergebrachtes und bewährtes Mittel zur kontrollierten Ableitung von Wundflüssigkeit, wobei insbesondere bei einliegenden Implantaten wegen der damit verbundenen Fremdkörperwirkung im Sinne von Sekretbildung diese als Dauerdrainage bis zur Metallentfernung ausgelegt sind.

Das Management der genannten Techniken zur Therapie der Infektkomplikation soll in der Form dargestellt werden, dass die geeignete Indikation, eine Art Gebrauchsanweisung bei der Durchführung dieser Verfahren nicht zuletzt aber auch Kontraindikationen und mit ihrem Einsatz verbundene mögliche Probleme erläutert werden. In diesem Zusammenhang darf der Hinweis nicht fehlen, dass alle diese Maßnahmen auf die jeweils individuellen Gegebenheiten abzustimmen sind und dass bei zu erkennender Unwirksamkeit alternative therapeutische Optionen ausgewählt werden müssen.

Die beschriebenen Behandlungsmöglichkeiten der Vakuumversiegelungstechnik, der Jet-Lavage, der lokal anzuwendenden Antibiotikaträger, der Kunsthautdeckung und der Wunddrainagen stellen in jedem Fall nur Bausteine im Konzept der Infektsanierung dar, wobei der chirurgischen Revision stets das Primat gebührt.

\section{Vakuumversiegelung}

Oberstes Behandlungsprinzip der Wundbehandlung ist, die Pathophysiologie zu erfassen bzw. letztere kausal zu behandeln. Dadurch ist der Grundstein für jede erfolgreiche Wundbehandlung gelegt und die Heilung kann durch moderne unterstützende Behandlungsverfahren beschleunigt werden.

Dabei hat sich in den letzten 10 Jahren die Wundbehandlung mit Vakuumtechnik derart etabliert, dass eine stetige Erweiterung der Indikation resultierte.

Bei drohenden und manifesten Infekten nach Osteosynthese oder Endoprothese steht die zeitnahe Revision als erste und wichtigste Maßnahme im Vordergrund.

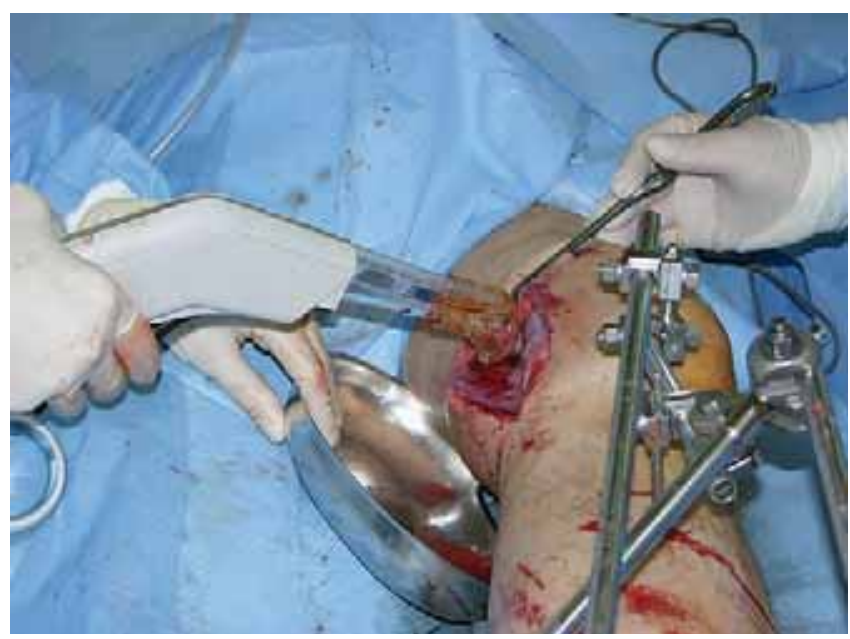

Abb. 2 Weichteilbefund der Fraktur mit verschmutztem Decollement des distalen Ober- und Unterschenkels.

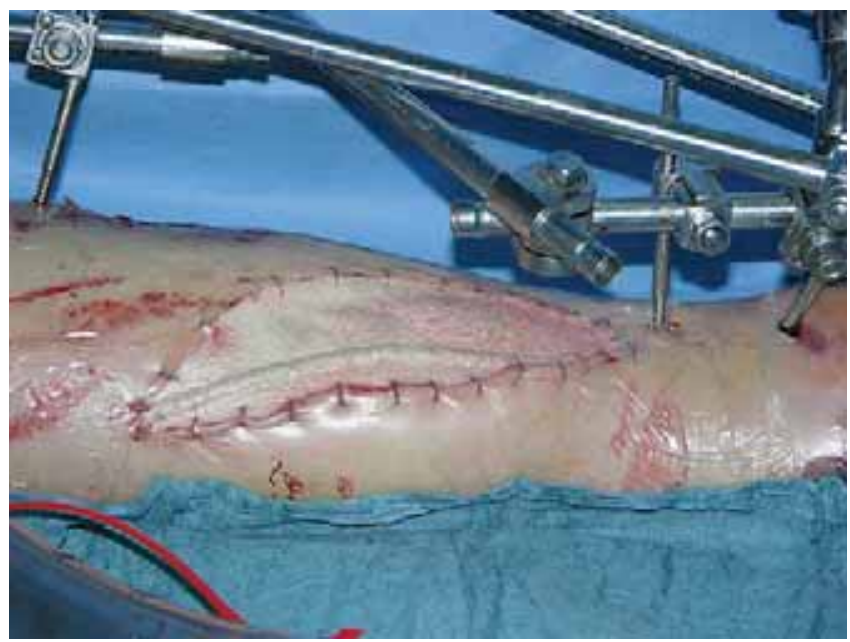

Abb. 4 Vergrößerung des distalen Unterschenkels.

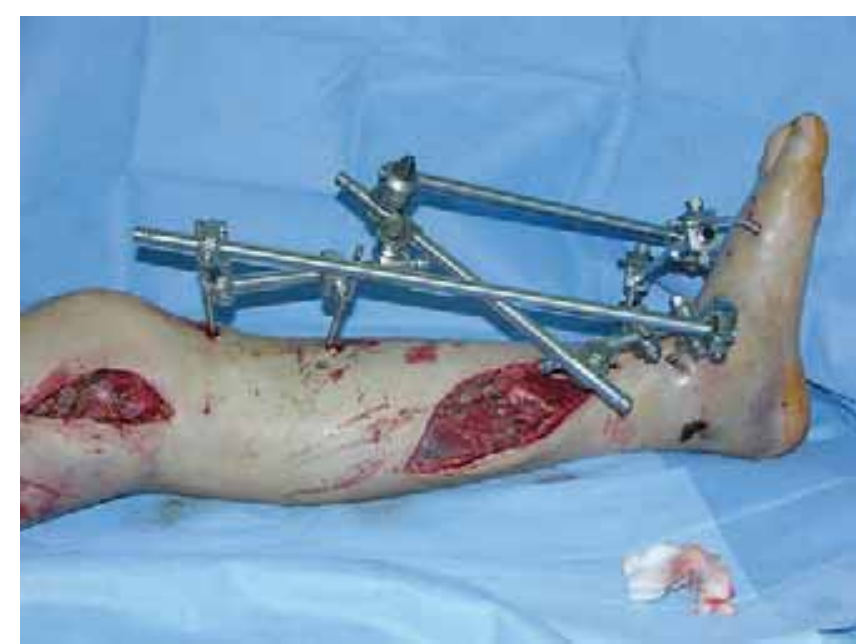

Abb. 3 Jet-Lavage zur effizienten Weichteil- und Knochenspülung.

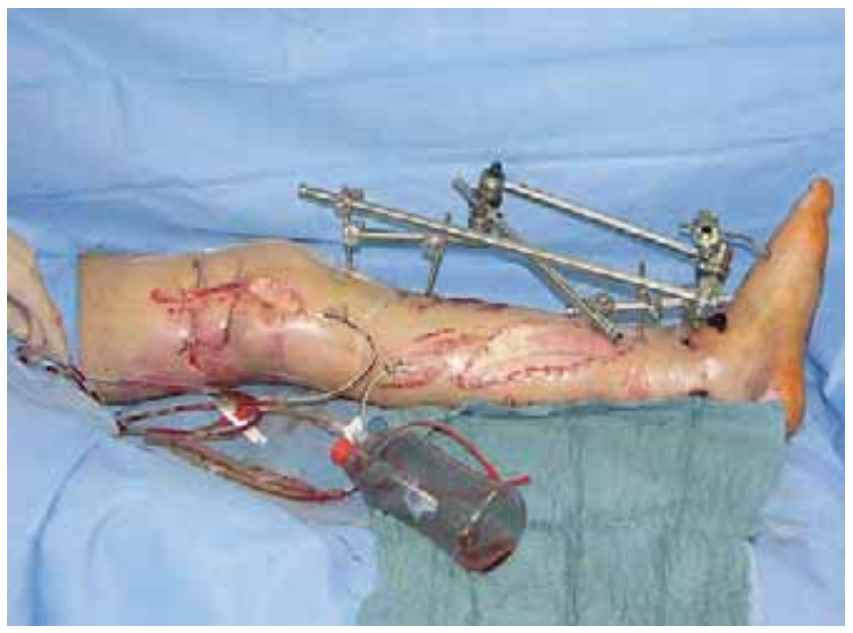

Abb.5 Vakuumversiegelung der Weichteildefekte zur weiteren Konditionierung, um bei erzielter Granulation die Spalthautdeckung auszuschließen. 
Intraoperativ wird ein Abstrich entnommen zur mikrobiologischen Aufarbeitung gefolgt von einem möglichst radikalen Debridement, wobei zum einen die Dekontamination und zum anderen die Verbesserung der lokalen Durchblutung angestrebt wird. Die intraoperative Wundspülung ist eine weitere sehr wichtige Maßnahme, um das potenziell mit Bakterien kolonisierte Implantat zusätzlich zu dekontaminieren. Anschließend sollte das gesamte OP-Gebiet mit einer suffizienten Drainage versorgt werden, damit sich keine Flüssigkeitsverhalte bilden, welche einen Infekt und eine Infektbegleitreaktion der Weichteile unterhalten können.

Mit der seit ca. 10 Jahren bestehenden Vakuumversiegelungstechnik steht dem Chirurgen ein sehr nützliches Werkzeug zur Verfügung, da postoperativ offen oder halboffen zu behandelnde Wunden verschlossen werden können, wodurch dem Patienten eine angenehmere Wundbehandlung geboten wird [1,5]. Das notwendige Lockern herkömmlicher Drainagen zur Gewährleistung der Funktion über 3 Tage hinaus entfällt. Bei der Vakuumtherapie wird ein grobporiger Schwamm aus Polyvinylalkohol oder Polyurethan in die Wunde eingelegt. Dieser ist mit einem Redonschlauch (vorzugsweise 12 - 16 Charrière) armiert, wobei die Perforationen nur im Schwamm liegen sollen. Die Wunde wird daraufhin entweder versiegelt (mit einer durchsichtigen Folie) oder mit Nähten verschlossen.

Bei Entschluss zu Versiegelung wird die Haut mit Waschbenzin entfettet, die Folie bei einliegendem Schwamm über der gesamten Wunde luftdicht verklebt und der Sog entweder über eine herkömmliche Redonflasche oder über eine Unterdruckpumpe anlegt.

Das Kleben der Folie sollte sowohl auf der Wunde als auch um die Wunde herum faltenfrei erfolgen, da sonst der Unterdruck nicht sicher aufrechterhalten werden kann.

In der Praxis hat sich ein ausreichend großer Abstand der Redon- bzw. Drainageaustrittsstelle bewährt, da es sonst zu einem Luftleck mit Verlust des Unterdruckes kommt. In solchen Fällen kann man auf eine Unterdruckpumpe ausweichen. Man wählt einen Unterdruck von ca. 75 bis $150 \mathrm{mmHg}$ [9]. Falls sich das Leck wundnah befindet und/oder Luft durch die Wunde gesaugt wird, empfehlen wir diese Situation zeitnah zu beheben, das heißt die Versiegelung neu anzulegen oder bei lokal schwierigen Weichteilbedingungen alternativ von dieser Therapieform abweichen, um die offene oder halboffene Wundbehandlung durchzuführen. Solche Situationen sind eher selten und können teilweise umgangen werden, indem man bei liegendem Fixateur externe die Pinstellen so wählt, dass die Folie nach Versiegelung außerhalb des OPs gewechselt werden kann.

Der Nachteil der Vakuumversiegelung mittels Folie ist ein größerer Kontrollaufwand postoperativ, da Flüssigkeitsverhalte zwischen Folie und Schwamm die Wundverhältnisse durch Mazeration verschlechtern können. Zudem muss man in solchen Fällen von einem Drainagepro- blem in der Wundtiefe ausgehen. Der zweite nachteilige Aspekt kann durch eine längere Wundbehandlung mit Vakuumversiegelung vor allem bei dünnen und vulnerableren Weichteilen wie z.B. in der Fuß- und Sprunggelenksregion, aber auch anderen Lokalisationen mit ähnlicher Weichteilqualität, entstehen. Es kommt zur Retraktion des gesamten Weichteilmantels mit der möglichen Entstehung eines fixierten Weichteildefektes, wobei koinzident narbig eingezogene Wundränder den Verschluss zusätzlich erschweren können. Solche Prozesse sind nicht nach einer Woche Behandlungsdauer zu erwarten, man sollte dies jedoch vor allem bei langer Behandlungsdauer im Hinterkopf behalten.

Als Alternative zur Versiegelung kann die Vakuumwundbehandlung bei verschlossenem Weichteilmantel erfolgen.

Der Wundschwamm wird in die Wunde bzw. Infektregion eingelegt, die Weichteile werden z.B. mit durchgreifenden Fäden (alternativ auch Fasziennähte) und Hautnähten primär verschlossen. Die Beurteilung der suffizienten Wunddrainage muss indirekt durch postoperative Wund- und Laborkontrollen erfolgen. Aus Erfahrung empfehlen wir den Weichteilmantel während der Vakuumwundbehandlung über durchgreifende Nähte zu verschließen, um die oben erwähnte Weichteilproblematik mit resultierenden Defekten zu vermeiden.

Nach ca. 5 bis 10 Tagen, in der Regel nach 7 Tagen, empfiehlt es sich, die Schwämme zu entfernen und ggf. durch frische zu ersetzen.

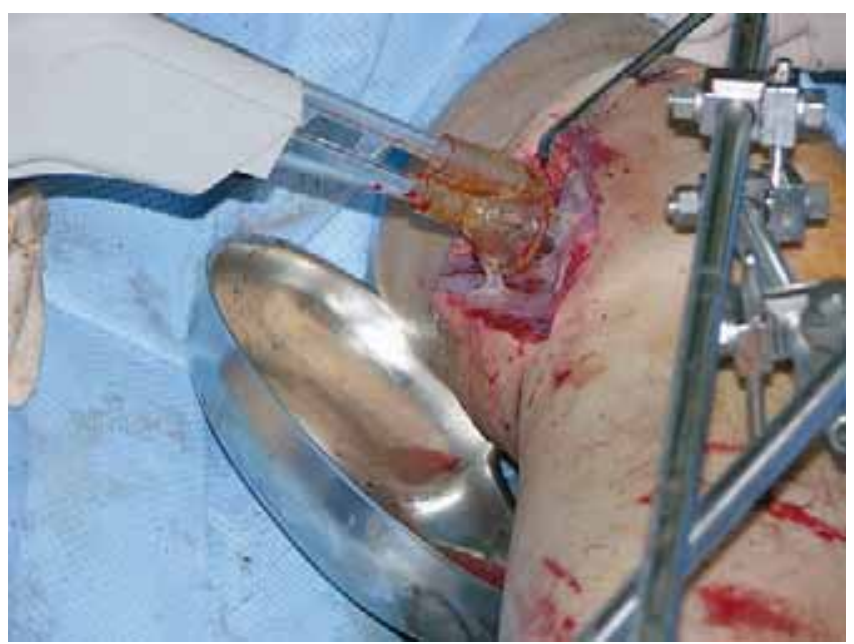

Abb. 6 Jet-Lavage einer verschmutzten Weichteildecollement-Verletzung des distalen Oberschenkels.

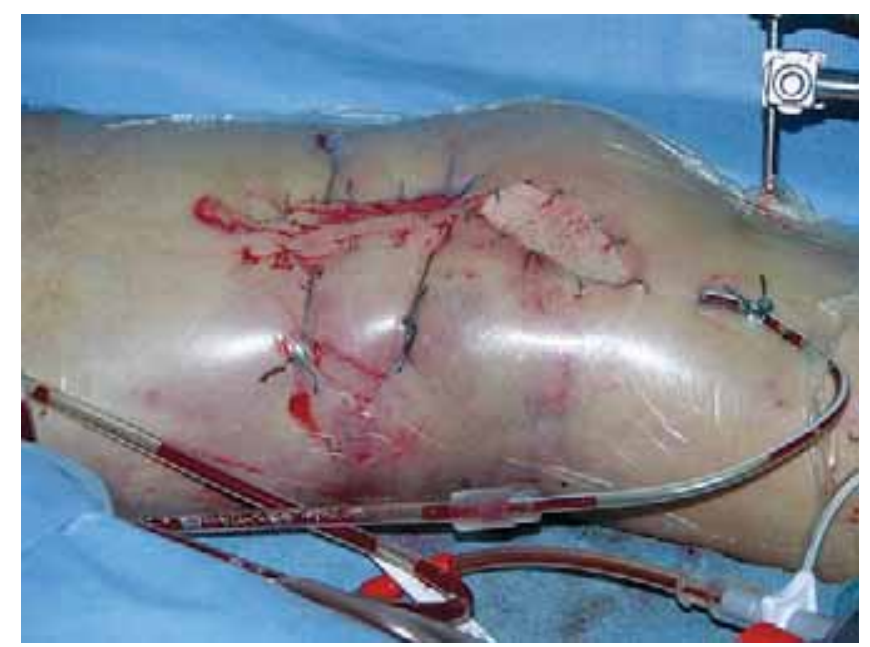

Abb.7 Vergrößerung bei partiellem Verschluss des Defektes in Kombination mit einer Vakuumversiegelung. 
Falls Wundschwämme länger als 10-12 Tage in situ verbleiben, kommt es zum Einwachsen der Weichteile, so dass die Entfernung mit jedem weiteren Tag schwieriger und traumatisierender wird.

Bei einer erfolgreichen Behandlung zeigt sich in der ehemaligen Infektregion eine gute Granulation mit zurückgebildeter Umgebungsrötung und Schwellung. Durch diesen Effekt hat sich die Vakuumversiegelung bzw. Vakuumtherapie in den letzten Jahren etabliert und eine stetige Indikationserweiterung erfahren, so dass auch chronische Wunden mittlerweile zum Einsatzbereich gehören [5]

Beispielhaft sind folgende Kontraindikationen zur Vakuumversiegelungstechnik oder Vakuumtherapie zu nennen:

- infizierte maligne Tumoren

- Fisteln zum Gastrointestinaltrakt

- freiliegende und somit arrosionsgefährdete Gefäße, inklusive Lymphfisteln (am häufigsten in der Leiste)

- Anaerobierinfekte (Gasbrand als Maximalvariante)

- nekrotisierende Fasziitis

Als besondere Einsatzindikation ist der Vollständigkeit halber auch das chronische Ulcus cruris zu nennen. Die koinzident bestehende Ausprägung der Dermatofasziosklerose muss unbedingt Berücksichtigung finden, da zusätzlich eine ausgedehnte Fasziektomie (partiell bis subtotal) Grundvoraussetzung für die erfolgreiche Vakuumtherapie ist. Diesem Krankheitsbild liegt ein erhöhter Gewebsdruck zugrunde. Hier ist die Versiegelung mit der Folie erforderlich.

Die Versiegelung in Sandwichtechnik nach erfolgter Spalthautdeckung kann
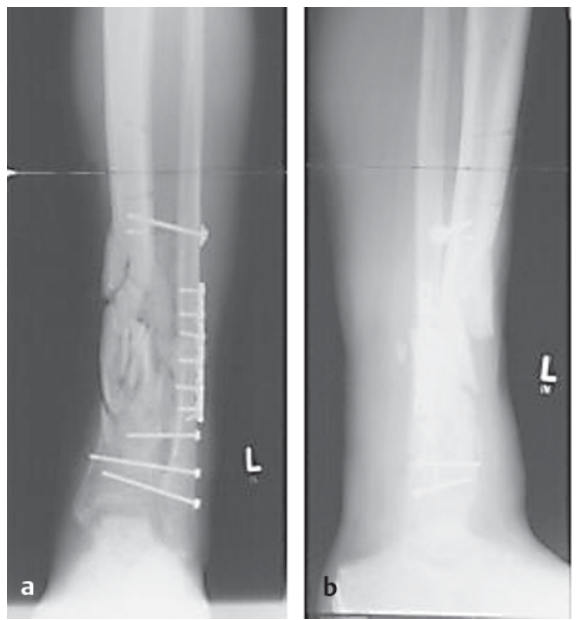

Abb. 8 Röntgenverlaufsserie
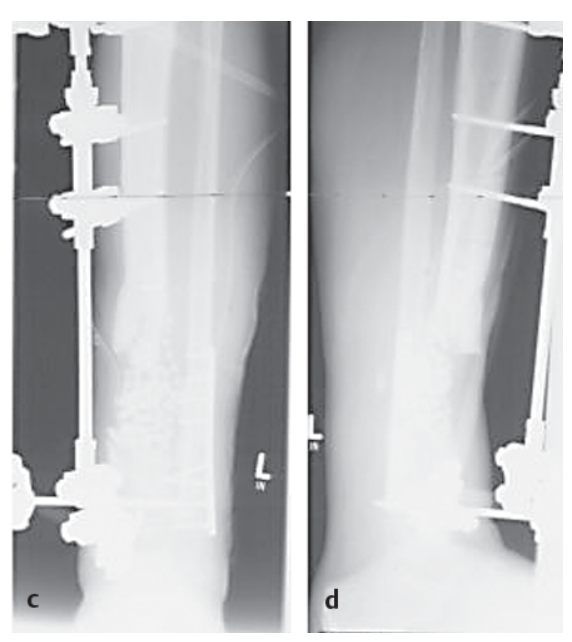

bei stark sezernierender Wunde zur Fixierung des Transplantates und/oder zur verbesserten Drainierung genutzt werden, damit das Transplantat sicher angeht.

Zusammenfassend steht die chirurgisch kausale Therapie bei Infekten von Knochen mit und ohne Osteosynthesen sowie Endoprothesen unstreitbar an erster Stelle, wobei die Vakuumwundtherapie zu einer Beschleunigung der Restitution führen kann, bedingt durch eine geometrisch erhöhte Drainagekapazität.

Gleichzeitig wird die granulative Reaktion von Knochenoberflächen wie Weichteilen gefördert, so dass eine Sekundärnaht mit zeitnahem definitivem Wundverschluss möglich ist.

\section{Jet-Lavage}

Die allgemeinen Prinzipien der Infektbehandlung auf chirurgischem Gebiet haben sich über viele Jahrzehnte bewährt, wobei sich die zur Verfügung stehenden technischen Hilfsmittel stetig weiterentwickelt haben. Diese Entwicklung schloss auch die Spültechniken mit ein, wobei neben der normalen Spülung mit einer Blasenspritze oder einer Kanne die Jet-Lavage als Alternative zur Anwendung kam.

Prinzipiell kann man zwischen intra- und postoperativer Spülung bzw. Lavage unterscheiden. Zusätzlich stehen Niedriggepulste Modulation zur Verfügung. Es gibt unterschiedliche kommerzielle Anbieter. Bei der postoperativen Spülung wird hauptsächlich die Spül-Saugdrainage eingesetzt. und Hochdruckspülungen mit und ohne
Neben der Spültechnik ist die Spülflüssigkeit zu erwähnen, wobei die Pathophysiologie und die anatomischen Besonderheiten der betroffenen Region bei der Wahl der Spülflüssigkeit eine entscheidende Rolle spielen.

Die gängigste seit Jahrzehnten etablierte intraoperative Spülung wird mit der so genannten „Blasen“-Spritze durchgeführt. Diese hat ein Volumen von ca. $100 \mathrm{ml}$ und ihren Ursprung in der Urologie, wobei der Anwender den Druck im wahrsten Sinne des Wortes selbst in der Hand hat.

Um die Spülung zu verbessern, wurden elektrisch betriebene Spülsysteme entwickelt, die entweder einen kontinuierlichen oder intermittierenden so genannten gepulsten Strahl aufbringen. Die unterschiedlichen Drücke lassen eine Einteilung in Hoch- und Niedrigdrucksysteme zu.

In den letzten 10 Jahren hat sich die gepulste bzw. pulsatile Hochdruck-Lavage, die wir im klinisch umgangssprachlichen Gebrauch als „Jet-Lavage“ bezeichnen, durchgesetzt.

Neben den unterschiedlich stark ausgeprägten bakteriziden Eigenschaften der Spülflüssigkeit ist hauptsächlich der Druck für die bakterielle Clearance verantwortlich mit der damit verbundenen Spüldauer und Spülvolumen.

Unterschiedliche In-vitro-Studien haben gezeigt, dass eine direkte Beziehung zwischen der bestehenden Kontaminationszeit mit der Adhärenz der Bakterien (z.B. Staphylococcus aureus) am Knochen besteht. Bei einer experimentell erzeugten bakteriellen Kontamination von 3 Stunden ist der dekontaminierende Effekt einer normalen Spülung genauso einzuschätzen wie derjenige der pulsatilen Jet-Lavage.

Nach 6 Stunden findet man eine objektivierbare Überlegenheit der bakteriellen Clearance durch die Anwendung der JetLavage gegenüber der herkömmlichen Spülung [1].

Zusätzlich ist die mikroskopisch nachweisbare Traumatisierung sowohl des Knochens als auch der Weichteile zu berücksichtigen. Diese ist im Vergleich zur herkömmlichen Spülung bei der JetLavage signifikant stärker ausgeprägt, so dass man insbesondere bei angestrengten Weichteilen, sei es nach einem Trau- 


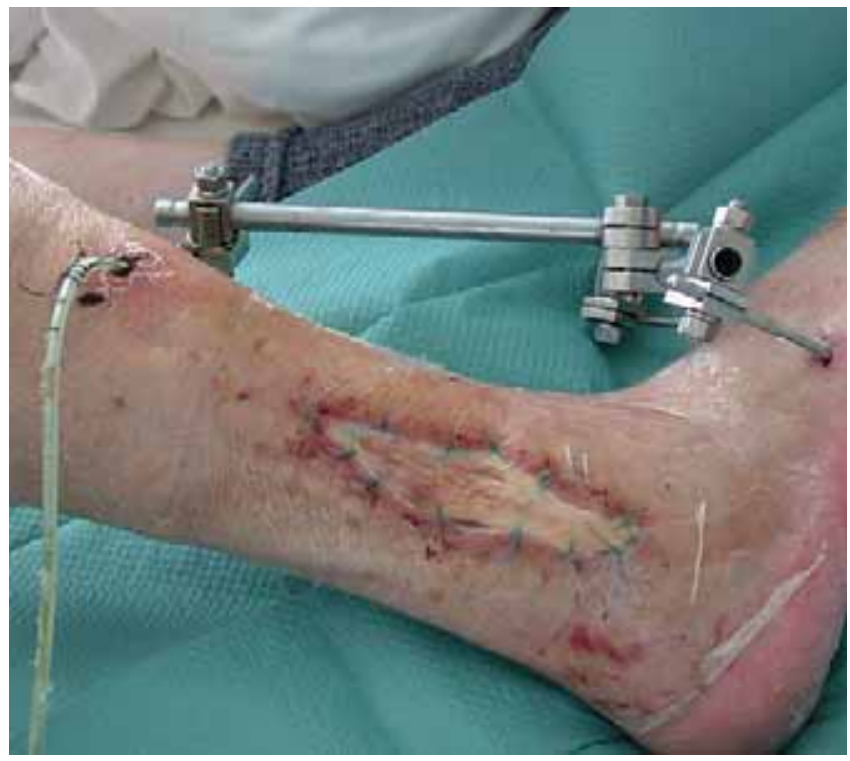

Abb.9 65-jähriger Patient mit pAVK und Nekrosen über einer Außenknöchelplatte nach bimalleolärer Sprunggelenksluxationsfraktur. Nach Nekrektomie, Metallentfernung und Fixateuranlage wurde der Weichteildefekt versiegelt. Zusätzlich wurde eine Dilatation der artiellen Unterschenkelstrombahn durchgeführt. Zur Verhinderung einer Wundranddistraktion wurde der PVA-Schwamm eingenäht.

ma oder bei einem akuten (z.B. einschmelzendem) Infektgeschehen den Einsatz der Jet-Lavage überdenken muss. Einen weiteren Aspekt der JetLavage sollte man an dieser Stelle nicht unerwähnt lassen: Durch die mechanisch gesehen aggressivere Spülung ist ein $\mathrm{Mi}$ krodebridement möglich, welches die chirurgische Behandlung zusätzlich optimiert.

Die Wahl der Spüllösung kann bei der Fülle unterschiedlicher Substanzen mitunter schwierig sein. Bei implantatassoziierten Infekten ist der Einsatz von Lava$\operatorname{sept}^{\circledR} \mathrm{zu}$ bevorzugen. Es empfiehlt sich insbesondere bei Protheseninfekten mit 2-3 Litern zu spülen. Die Einwirkzeit von 3 Minuten ist zu beachten. Danach spült man mit physiologischer Kochsalzoder Ringer-Lösung. Bei Gelenkinfekten ohne Gelenkersatz darf Lavasept ${ }^{\circledR}$ nicht genommen werden, um den Knorpel nicht zu schädigen.

Bei septischer Kreislaufsituation mit Katecholaminpflichtigkeit des Patienten sollte unseres Erachtens nach die Jet-Lavage unterbleiben, da durch sie das Einschwemmen von Bakterien in den systemischen Kreislauf resultieren kann, wodurch der Patient bei instabiler Ausgangssituation gefährdet wird.

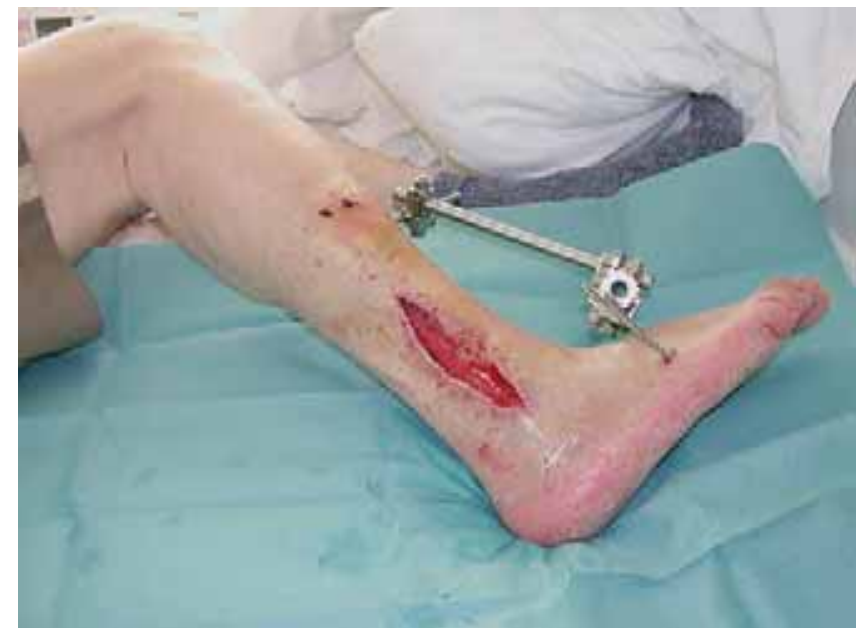

Abb.10 Der ehemalige nekrotische Defekt ist gut mit Granulationsgewebe ausgefüllt, die distale Fibula ist gut bedeckt. Es liegt Infektfreiheit vor.

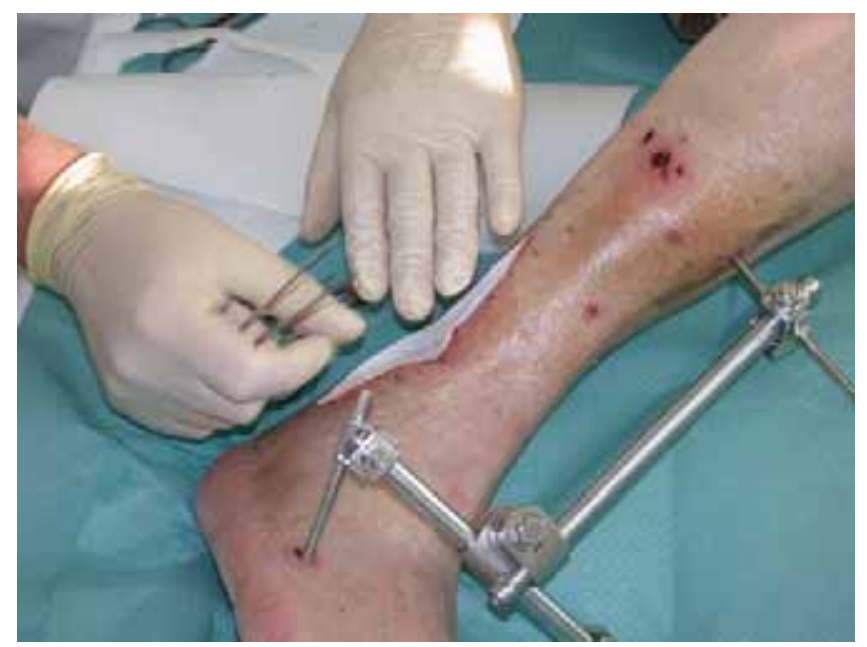

Abb.11 Exaktes Einpassen von Epigard ${ }^{\circledR}$ als temporärer Hautersatz bis zur Spalthautdeckung.

Aus dem oben gesagten ergibt sich somit ein relativ großes Einsatzgebiet:

Im aseptischen Bereich ist hauptsächlich die verbesserte Knochenzementinfiltration in die spongiöse Knochenarchitektur zur Implantation von Gelenkprothesen zu nennen, welche durch mehrere In-Vitro-Studien bewiesen wurde [5].

Im septischen Einsatzbereich der Jet-Lavage sind vor allem Früh- bzw. Akutinfekte nach Prothesenimplantation zu nennen. Bei Osteitis und Osteomyelitis hat sie vor allem im akuten Stadium mit Beteiligung sowohl kortikaler als auch spongiöser Knochenanteile eine wichtige Bedeutung. Bei chronischen Infektprozessen des Knochens mit sklerosierter Knochenqualität steht das knöcherne Debridement zur Infektbeherrschung im Vor- dergrund, gepaart mit einer testgerechten antibiotisch unterstützenden Medikation, ggf. mit einer zusätzlichen myoplastischen Operation, um die lokale Durchblutungsqualität zu verbessern.

Akute Verletzungen mit kontaminierten bis verschmutzen Knochen- und Weichteilen (vor allem offene Frakturen) dürfen nicht vergessen werden.

Die Erfahrung des Operateurs bei der Beurteilung der Weichteile spielt eine wichtige Rolle, da die traumatisierenden Effekte der Jet-Lavage nicht vergessen werden dürfen.

Einige Arbeiten empfehlen trotzdem den großzügigen Einsatz bei akuten offenen Knochen- und Weichteilverletzungen. 


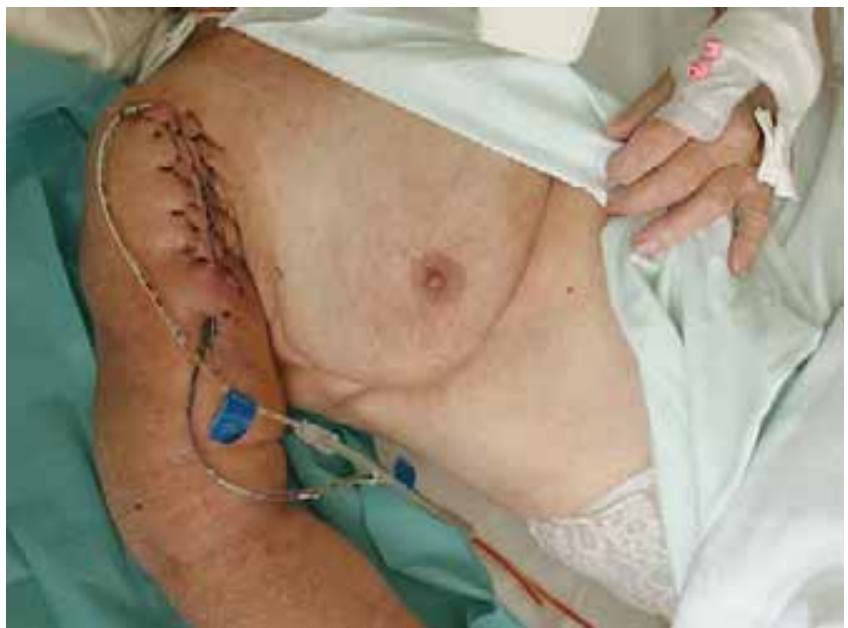

Abb.12 Einliegende Redondrainagen nach Hämatomrevision bei einer 78-jährigen Patienten und Leberzirrhose Child B. Die Drainagen liegen im Plattenlager nach Metallentfernung einer proximalen Humerusplatte.

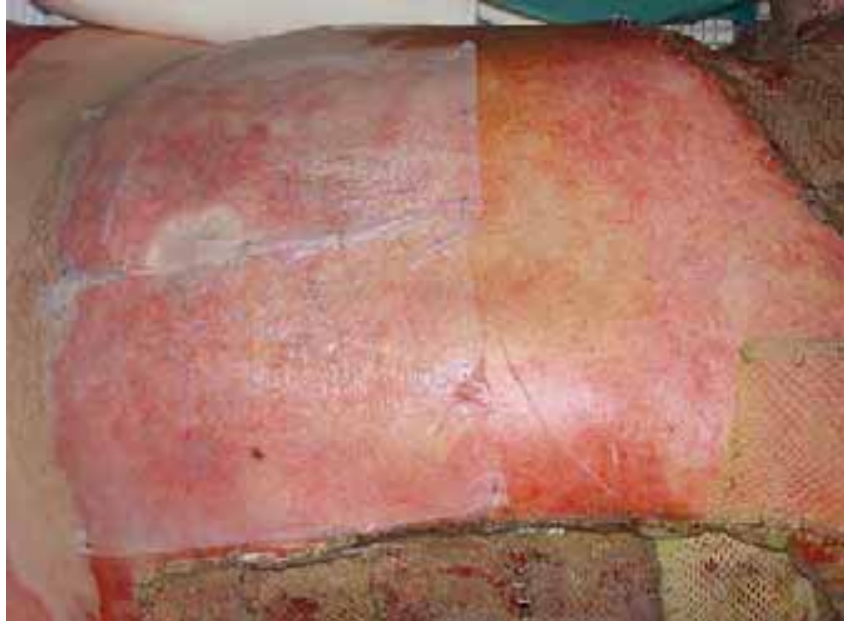

Abb.13 Rumpfverbrennungswunden, die temporär mit Biobrane ${ }^{\circledR}$ gedeckt wurde. Hierbei handelt es sich um ein Silikon-Nylon-Netz, das mit porcinem Kollagen beschichtet ist.
Zusammenfassend überwiegen die Vorteile der Jet-Lavage und bieten auf unfallchirurgisch-orthopädischen Gebieten ein zusätzliches effektives Instrument zur Infektbeherrschung. Insbesondere bei akuten Ereignissen wie frischen Protheseninfekten, akuter Osteitis und Osteomyelitis nach Fraktur mit osteosynthetischer Versorgung und offenen Frakturen ist die Jet-Lavage indiziert.

\section{Mikrobiologische Proben}

Das umgehende und subtile chirurgische Debridement ist bei der Behandlung von Infektionen in der Knochen- und Gelenkchirurgie unumgänglich.

Die Keimdiagnostik - unter sterilen Bedingungen - ist dabei Grundvoraussetzung, um eine präzise antimikrobielle Therapie zu beginnen.

Entzündungen des muskuloskelettalen Bereichs und insbesondere die Fremdkörper-assoziierten Infektionen sind in der Regel durch eine niedrige Keimzahl in den Proben und eine schwierige Abgrenzung von Kontaminanten der normalen Hautflora charakterisiert. Folglich muss ein großes Probenvolumen gewonnen werden, welches repräsentativ für die Lokalisation des Infektgeschehens und wenig kontaminationsträchtig ist. Fistelabstriche, Analysen von Drainageflüssigkeiten und oberflächliche Wundabstriche sind aufgrund der hohen Kontaminationsgefahr zu vermeiden. Am häufigsten werden Ergussflüssigkeiten bei Gelenkinfekten sowie Abstriche und besser noch intraoperativ gewonnene, repräsentative Gewebeproben aus meh- reren verdächtigen Regionen zur Untersuchung herangezogen.

Bei Infektionen mit einliegendem Implantat sollten die Gewebeproben aus der Grenzschicht zum umgebenden Gewebe stammen und mehr als $1 \mathrm{~cm}^{3}$ betragen $[6,7]$.

Die Proben werden in entsprechenden Transportmedien gelagert und möglichst rasch in das mikrobiologische Labor verbracht. Hier kann eine erste, noch unspezifische Aussage über das Vorliegen einer Infektion durch eine Gramfärbung gemacht werden. Die weitere Bebrütung erfolgt in der Regel für 2-4 Tage. Falls sich danach kein Nachweis bietet, ist die kulturelle Anzüchtung für 10-14 Tage nötig, um gerade bei Low-Grade-Infektionen der kleinen Keimzahl und der langen Verdoppelungszeit der Erreger gerecht zu werden. An die Isolierung des Keims schließt sich die Identifizierung und Resistenzprüfung an. Auf der Grundlage des Erregernachweises ist dann eine spezifische Therapie möglich.

\section{Antibiotikaträger}

Bei der Behandlung von infizierten Pseudarthrosen und der chronischen Osteomyelitis sind neben der Beherrschung der Infektsituation auch die Weichteildeckung und die Herstellung der knöchernen Heilung das Therapieziel. Die Infektionskontrolle basiert dabei auf einem radikalen, chirurgischen Debridement und einer dem jeweiligem Keimspektrum angepassten Therapie mit einem Antibiotikum.
Die Schlüsselelemente der lokalen Antibiotikatherapie sind zum einen das $\mathrm{Ab}$ gabevehikel und zum anderen das inkorporierte Antibiotikum.

Bei den Abgabevehikeln werden die biologisch abbaubaren von den häufig verwendeten nicht biologisch abbaubaren Substanzen unterschieden. $\mathrm{Zu}$ diesen gehören der PMMA-Zement, wie er aus der Endoprothetik bekannt ist. Der Knochenzement kann dabei als Spacerblock oder als Kugeln aufgereiht auf einem chirurgischen Draht in unterschiedlicher Anzahl $(10 / 20 / 30 / 60)$ und unterschiedlicher Größe (ca. 5-10 mm) geliefert werden.

Zu den biologisch abbaubaren Trägersubstanzen zählen u.a. Kollagenschwämme, Keramik- und Hydroxylapatitblöcke, Polylaktit/Polyglykolit-Implantate, Fibrin und Nahtmaterial.

Eine nicht unbeträchtliche Anzahl von antimikrobiell wirksamen Substanzen kann erfolgreich in Knochenzement verarbeitet werden. Hierzu gehören Aminoglykoside, Vancomycin, Cephalosporine und Chinolone.

Drei Grundvoraussetzungen müssen erfüllt sein:

1. Das Antibiotikum muss als Pulver zur Verfügung stehen, damit es gut mit den PMMA-Partikeln vermischt werden kann.

2. Die Substanz muss hitzestabil sein, damit beim Polymerisationsprozess des Knochenzements nicht ihre Wirkung verliert.

3. Das Antibiotikum muss wirksam gegen den nachgewiesenen Keim sein. 
Falls vor der Operation mikrobiologische Kulturergebnisse und entsprechende Resistenztestungen vorhanden sind, kann das richtige Antibiotikum zugeordnet werden. Andererseits sollte das lokale Antibiotikum den häufigsten Keimen angepasst werden. Dies sind im Falle der chronischen Osteomyelitis Staphylococcus aureus in ca. 65-70\% und Pseudomonas aeruginosa in 20-37\% der Fälle. Die gebräuchlichsten Medikamente sind Aminoglykoside und Vancomycin.

Die Abgabe des Antibiotikums hängt hauptsächlich von der Trägersubstanz, dem Antibiotikum selbst, der Umgebung und der Zeit nach der Implantation ab.

Bei offenen und drainagenversorgten Wunden sollten die lokalen Antiobiotikaträger nicht verwendet werden, da keine wirksame Konzentration am Ort des Infekts erreicht wird [13].

Die lokale Therapie mit Antibiotika wurde durch Buchholz und Engelbrecht eingeführt. Sie konnten dabei nachweisen, dass bei der chirurgischen Behandlung von frühen postoperativen Protheseninfektionen die Infektrate reduziert werden kann. Dabei wird dass Antibiotikum in Polymethylmethacrylat (PMMA)-Zement gemischt, in den sanierten Infektherd eingebracht und erreicht dort eine hohe lokale Konzentration, wobei gleichzeitig die systemische toxische Wirkung reduziert wird [4]. Nachfolgend wurden dieses Vorgehen auch bei der Behandlung der Osteomyelitis und offener Frakturen angewendet.
In unserer Klinik kommen die lokalen Antibiotikaträger vor allem bei Behandlung der Osteomyelitis zum Einsatz, bei der nach radikalem chirurgischem Debridement Defektzonen entstanden und weitere Operationen geplant sind. Sie eigenen sich dabei hervorragend als additives Therapeutikum mit Spacerfunktion, bis sie im Rahmen der Sekond-look-Operation, der Weichteildeckung mit Lappen oder der Spongiosplastik nach einer bis sechs Wochen wieder entfernt werden.

\section{Kunsthautdeckung}

Der klinische Bedarf an Hautersatzmaterialien zum Verschluss akuter oder chronischer Wunden ist nach wie vor groß, da trotz intensiver Forschung in der Wundheilung die dermale Regeneration ein Problem darstellt. Trotz aller Erfolge in der Plastischen Chirurgie basieren alle plastischen Methoden auf dem Vorhandensein von ausreichend Eigengewebe. Gerade bei ausgedehnter Traumatisierung (z.B. thermische Schädigung) ist dieses oft nur in begrenzter Menge verfügbar [8].

Die Entwicklung von Hautersatzmaterialien lässt sich in zwei Schwerpunkte untergliedern. Zum einen werden Produkte entwickelt, die ein Heilungsmilieu garantieren, so dass körpereigene Reparaturmechanismen greifen können. Der zweite Entwicklungsschwerpunkt betrifft echte Hautanaloga, die als Alternative zur Spathauttransplantation angeboten werden.

Die Anwendung von Hautersatzmaterialien kann passager oder permanent sein, die Herstellung autologe Komponenten (Matrixsysteme) enthalten oder rein synthetisch erfolgen [14].
Folgende Anforderungen werden an Hautersatzmaterialien gestellt werden:

- Barrierefunktion zur Verhinderung einer Wundinfektion

- Immunologische Verträglichkeit

- Sicherstellung der Thermo- und der Flüssigkeitsregulation

- Einfache Applizierbarkeit

- Sicherstellung des Remodelings und Beschleunigung der Wundheilung

- Adäquates Preis-Leistungs-Verhältnis

- Unbegrenzte Verfügbarkeit mit problemloser Lagerungsfähigkeit.

Neben der Unterteilung in temporäre und permanente Hautersatzverfahren können sie bezüglich ihrer Materialeigenschaften unterschieden werden:

- rein biologisch

- synthetisch

- bioartifiziell im Sinne von Matrixsystemen.

Der Anspruch des jeweiligen Hautersatzverfahrens muss sich an den Wundtypen orientieren. Akute traumatische Wunden stellen bezüglich Perfusion und Oxygenierung kein relevantes Problem dar, so dass ein Wundmilieu geschaffen werden kann, das eine ungestörte Granulation garantiert.

Die chronische, septische Wunde ist durch Überschuss an körpereigenen (Metalloproteinasen) und bakteriellen Proteinasen gekennzeichnet. Diese Proteinasen können den regenerativen Prozess derartig stören, dass die Wunde sich selber unterhält (Chronizität). Dies muss bei der Wahl des entsprechenden Ersatzverfahrens berücksichtigt werden. Stark sezernierende Wunden beinhalten das Risiko der Umgebungsmazeration und sollten deshalb zunächst „trockengelegt" werden.

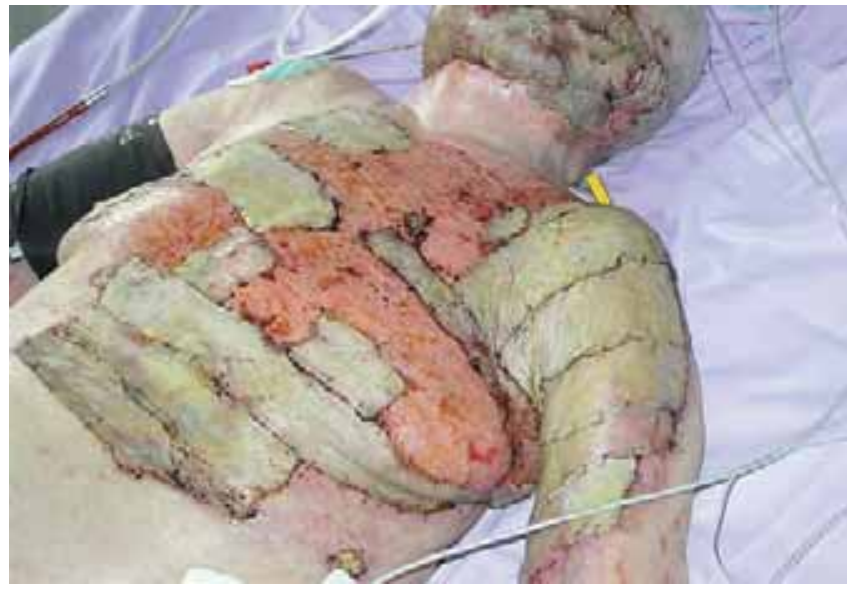

Abb.14 Hautersatz mit Euroskin ${ }^{\circledR}$ bei einem Verbrennungspatienten.

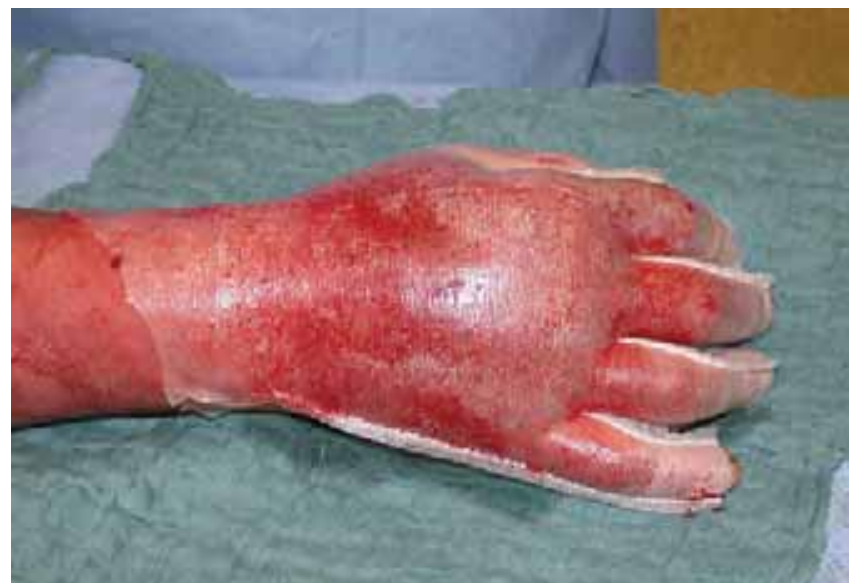

Abb.15 Temporärer Hautersatz mit Matriderm ${ }^{\circledR}$. Es ist ein Hautersatz, der aus unvernetztem, bovinem Kollagen besteht. 
Als sehr effizient haben sich Vakuumsysteme erwiesen, die in Kombination eines Schwammes und einer Polyurethanfolie funktionieren.

Erst nach Entstehung eines Granulationsrasens kann dann auf Hautersatzverfahren umgeschwenkt werden.

Als eines der ersten synthetischen Hautersatzverfahren wurde in den 70er-Jahren Epigard $^{\circledR}$ entwickelt. Hierbei handelt es sich um eine Polypropylenfolie, die mit einem Polyurethanschaumstoff beschichtet ist. Die Folie verhindert das Austrocknen der Wunde und bietet eine gute Barrierefunktion. Der Schaumstoff ermöglicht durch seine dreidimensionale Struktur das Wachsen von Granulationsgewebe, so dass sekundär eine erfolgreiche Spalthautdeckung möglich wird. Epi$\operatorname{gard}^{\circledR}$ eignet sich als temporäre Weichteildeckung bei offenen Frakturen, wobei hier eindeutig der Trend zur primären Weichteilversiegelung $\mathrm{zu}$ beobachten ist. Zudem ist Epigard ${ }^{\circledR}$ geeignet, Kompartmentwunden bis zum definitiven Weichteilverschluss zu bedecken. Epi$\operatorname{gard}^{\circledR}$ muss exakt in die Wunde eingepasst werden. Eine Fixierung ist mittels elastischer Wickelung, Steristrips und auch über Stapler möglich. Um ein Einwachsen des PU-Schaumes zu verhindern, sollte Epigard ${ }^{\circledR}$ täglich gewechselt werden.

Hydrokolloide (z.B. Comfeel ${ }^{\circledR}$ ) und Hydropolymere (z.B. Tielle ${ }^{\circledR}$ ) zeichnen sich durch ihre absorptiven Eigenschaften bezüglich der Wundsekretion aus, sind aber nur bei schwach oder mäßig exsudierenden Wunden geeignet. Sie sorgen für ein feuchtes Wundmilieu, das den Wundprozess unterstützt und optimale Bedingungen für die fortschreitende Granulation schafft. Hydrokolloide und Hydropolymere unterstützen zudem die Selbstreinigungskräfte der Wunde und sind vorteilhaft bezüglich des Proteinasenüberschuss. Der Verbandswechsel erfolgt je nach Exsudationsverhalten; der Verband kann auch mehrere Tage belassen werden und bietet so einen gewissen Vorteil bezüglich des Pflegeaufwandes. Bei stärkerer Exsudation der Wunde lassen sich Hydrokolloide mit Alginaten kombinieren, die unter zunehmender Flüssigkeitsabsorption eine gelartige Konsistenz annehmen.

Hydrogelverbände (z.B. TenderWet ${ }^{\circledR}$ ) werden vor Applikation mit z.B. Ringer-Lösung benetzt, um Wundstrukturen feucht zu halten. Sie sind ungeeignet bei sezer- nierenden Wunden. Hydrogel unterstützen durch das geeignete Wundmilieu die granulative Potenz. Durch die Anwendung von Hydrogelen lassen sich auch Wundbeläge lösen.

Perforierte Silikonauflagen (z.B. Mepithel $^{\circledR}$ ) können bei oberflächlichen Verletzungen angewendet werden. Durch die Perforationslöcher wird überschiessendes Exsudat in den Verband abgegeben. Silikonauflagen lassen sich gut wechseln, da eine Verklebung mit der Wunde nicht beobachtet wird. Im Gegensatz zu neutralfetthaltigen Gazen kommt eine Allergisierung nicht vor.

Als synthetisch-biologische Kombinationsprodukte werden zunehmend Folien aus Silikon oder Nylon mit Kollagenmatrizes oder Fibroblasten angeboten (Biobrane $^{\circledR}$, TransCyte $^{\circledR}$, Integra ${ }^{\circledR}$, Dermagraft $\left.^{\circledR}\right)$. Hierbei handelt es um einen temporären Hautersatz v.a. in der Verbrennungschirurgie.

Rein biologische Produkte als temporärer Hautersatz sind entweder allogenen oder xenogenen Ursprungs. Bei humanen Produkten handelt sich um dezellulierte Leichenhaut (AlloDerm ${ }^{\circledR}$ ), die gelegentlich auch mit Keratinozytensheeds kombiniert wird. Xenogene Transplantante sind reine Kollagenmatrices equiner (TissueFleece $\mathrm{E}^{\circledR}$ ) oder boviner (TissueFascie $^{\circledR}$ ) Herkunft.

Der permanente Hautersatz lässt sich unterteilen in die klassische autologe (Spalt-) Hauttransplantation und in die Transplantation von in der Zellkultur expandierten Keratinozyten. Als Standard gilt hier immer noch die Mesh-Transplantation. Trotz aller Bemühungen und Anstrengungen im Bereich des Tissue Engineerings sind bislang keine Transplantate verfügbar, die annähernd der normalen dermalen Funktion gerecht werden. Dies ist v.a. durch das Fehlen von Hautanhangsgebilden, durch mangelnde Elastizität und Sensorik bedingt. Auch die fehlende Pigmentierung dieser Transplantate ergeben oft nur ein unbefriedigendes ästhetisches Ergebnis. Man darf gespannt sein, ob die Stammzellforschung hier berechtigte Hoffnungen und Wünsche erfüllen vermag.

\section{Wunddrainagen}

Der Nutzen von Wunddrainagen in der Elektiv-, Notfall- und septischen Chirurgie ist unbestritten. Gegenstand der immerwährenden Diskussion ist die Frage, ob in jedem Fall eine Wunddrainage sinnvoll oder sogar verzichtbar ist [11]. Auch die Frage, ob eine Drainage per se ein Infektrisiko darstellt, ist trotz intensiver Studien bislang nicht schlüssig beantwortet worden [12].

Eine Drainage stellt ein Ableitungssystem dar, dass Körperflüssigkeiten nach außen ableitet oder erlaubt, Flüssigkeiten im Körper zu instillieren.

Wunddrainagen lassen sich prinzipiell nach ihrer Intention unterteilen:

1. reine Sekretdrainage

2. Indikatordrainage

3. Spüldrai nagen

Wunddrainagen bestehen aus Kunststoffen wie Polyethylen, Latex, Silikon und Gummi und werden perkutan, extravulnär ausgeleitet.

Die reine Sekretdrainage erlaubt ein kontinuierliches Ableiten von Blut und Wundflüssigkeit. Die Ansammlung von Blut und Wundflüssigkeit würde sonst zu einem Anstieg des lokalen Gewebedrucks führen und somit eine Perfusionsund Oxygenierungsstörung induzieren. Sekretdrainagen mit Unterdruck führen darüber hinaus zu einem rascheren Verkleben der Wundflächen, was wiederum zu einem Sistieren der Wundsekretion führt. Reine Sekretdrainagen werden so lange belassen, bis sich ein Gleichgewicht zwischen Wundsekretion und Sekretresorption eingestellt hat, was in der Regel nach zwei Tagen eintritt, so dass dann eine Wunddrainage entfernt werden kann. Unterdruckdrainagen (z.B. Redondrainage, Pleuradrainage) sind geschlossene Systeme und werden mit einem definierten Vakuum angelegt, um so einen kontinuierlichen Drainageeffekt zu gewährleisten. Sowohl das geschlossene Prinzip als auch der gerichtete Sog verhindert das Auftreten von aufsteigenden Infektionen. Das Vakuum wird durch Kunststoffflaschen mit definierten Sog und auch durch Pumpensysteme gewährleistet. Bezüglich des Unterdrucks muss eine Begrenzung vorhanden sein. Tierexperimentelle Studien konnten zeigen, dass ein zu großer Unterdruck Perfusionsschäden und somit Gewebeschäden verursachen kann.

Drainagen ohne definierten Unterdruck (z.B. Robinsondrainage) arbeiten nach dem Überlaufprinzip, wo der Drainagesog über das Druckgefälle und die Schwerkraft zwischen Drainageort und Sammelort aufrechterhalten wird. Wird 
der Drainageschlauch durch ein wassergefülltes System geführt, so arbeitet diese Drainage nach dem „Heber-Prinzip“.

Die Indikatordrainage findet vor allem in der Viszeralchirurgie ihre Anwendung, um rasch Komplikationen wie Blutungen und Insuffizienzen zu erkennen und $\mathrm{zu}$ behandeln. Gelegentlich wird die Drainageflüssigkeit laborchemisch untersucht, um die Herkunft einer Insuffizienz zu lokalisieren (Kreatininbestimmung bei Urinom, Bilirubinbestimmung bei Leckage der abführenden Gallenwege, Amylase bei Pankreasfistel, Triglyceride bei abdominellen Lymphfisteln, Gamfärbung bei Darminsuffizienzen, Hämoglobin in Zusammenschau mit dem Bluthämoglobin bei unklarer Blutung).

Prinzipiell bleibt bei dem Einsatz von Indikatordrainagen festzuhalten, dass die Quote von falsch negativen Beobachtungen nicht unbeträchtlich ist.

Spül-Saugdrainagen sind mehrlumig und erlauben neben der klassischen Wunddrainage auch Spüllösungen und Medikamenten zu instillieren. Sie finden ihre Verwendung v.a. in der septischen Chirurgie, um Abszesse oder Empyeme „auszutrocknen“. Durch die kontinuierliche Spülung soll verhindert werden, dass sog. Drainagekanäle entstehen und eine Drainierung einer Abszessformation oder eines Gelenks nur noch partiell gelingt. Als Spülflüssigkeit werden physiologische Spüllösungen, Antiseptika und Antibiotika verwendet. Auch Medikamente können über derartige Systeme incorporiert werden (z.B. bei rezidivierendem Pneumothorax, thorakale Lymphfisteln). Die Dauer einer Spül-Saugbehandlung wird vom klinischen Verlauf abhängig gemacht.

Saug-Spüldrainagen müssen täglich bilanziert werden.

Im Folgenden werden verschiedene Drainagetypen in der Weichteilchirurgie vorgestellt.

Die klassische Drainage ist die nach ihrem Entwickler benannte Redondrainage, die als Vakuumdrainage funktioniert. Intention dieser Drainage ist es, ein Wundhä- matom zu verhindern, um das Risiko einer Wundinfektion zu minimieren. Bei dieser Drainageform handelt es sich um ein geschlossenes System aus Einmalartikeln. Durch die relativen dünnen Lumina der Schläuche besteht das Risiko des Verstopfens der Schläuche durch Blut- oder Fibrinpfropfen, so dass ein längere Drainierung nur durch intensive Drainagepflege möglich ist. Gegenstand einer kontroversen Diskussion ist einerseits die These, dass durch die Drainage selbst einer aszendierenden Infektion Vorschub geleistet wird und andererseits, dass durch das kontinuierliche Vakuum der Blutverlust eher vergrößert wird. Eine eindeutige Studienlage zur Klärung dieser Fragen besteht bislang nicht. Methodisch hochwertige Studien lassen jedoch den Schluss zu, dass nicht in jedem Fall das Platzieren einer Wunddrainage einen Vorteil für den Patienten bringt. In Lokalisationen mit durch den elastischen Wundverband gut komprimierbaren Weichteilen und bei gründlicher Blutstillung ist sicherlich ein Teil der routinemässig eingelegten Wunddrainagen entbehrlich.

Ein wesentlicher Vorteil von Redondrainagen bei unvermeidbarem Blutverlust ist sicherlich die Rückführung des Wundblutes durch maschinelle Autotransfusion in der aseptischen Chirurgie.

Die Robinsondrainage aus Silikon findet ihre Anwendung in der Extremitätenchirurgie bei Amputationen als Überlaufdrainage, wo sie medial und lateral aus dem Amputationsstumpf ausgeleitet werden. Als Vakuumdrainage eignen sich Silikonschläuche nicht. Sie lassen sich durchaus als geschlossene Systeme führen, wenn auf das ausgeleitete Drainageende unter sterilen Bedingungen ein Sekretauffangbeutel aufgeklebt wird (halb-offenes System). Durch die Weichheit des Silikons besteht ein gewisser Vorteil bezüglich eines Arosionsblutungsrisikos im Vergleich zu PE-Materialien. Silikonschläuche haben dem gegenüber ein höheres Risiko abzuknicken und verlieren somit ihre Drainagewirkung.

Die Jackson-Pratt-Drainage besteht aus Silastik ${ }^{\circledR}$ und ist ebenfalls eine Vakuumdrainage, bei der das Vakuum über eine komprimierbares, ziehharmonikaähnliches Reservoir eingestellt wird.

Kapillardrainagen drainieren kleine, oberflächliche Wunden über den Kapillareffekt in die Wundauflage oder in einen Adhäsivbeutel. Typische Kapillardrainagen sind die einfache Gummilasche, die Penrosedrainage, die EasyFlow-Drainage und auch die so genannte Fadendrainage. Dieses Drainagentypen finden ihre Anwendung v.a. in der septischen Chirurgie.

Prinzipien der Drainagebehandlung

- dicklumige Drainagen sind vorteilhafter als dünnlumige

- weiche Drainagen haben ein geringeres Risiko einer Arosionsblutung

- Drainagenentfernung ohne Sog

- tägliche Dokumentation der Sekretmenge und -qualität

- Drainagen verhindern keine Infektion

- standardisierte Markierungen erleichtern die Lokalisation und Pflege - Vermeidung einer akzidentellen Drainagenfixierung durch die Wundnähte

\section{Schlussfolgerung}

Die praktische Durchführung von Behandlungsmaßnahmen bei akuter und chronischer Infektion in Unfallchirurgie und Orthopädie kann auf einige technische Maßnahmen zurückgreifen, die in der Palette der Behandlung von Entzündungskomplikationen unter verschiedener Indikation eingesetzt werden. Um eine möglichst zeitgerechte und umfassende Sanierung des Infektes zu erzielen, ist regelmäßig ein therapeutischer Stufenplan aufzustellen, in welchem die genannten Techniken ihren festen Platz haben. Sowohl der Nachweis der für die Infektion verantwortlichen Keime als auch deren gezielte medikamentöse und Lokalbehandlung basieren auf Erfahrungen und Gesetzmäßigkeiten, die ausführlich dargestellt worden sind. Dabei ist noch einmal hervorzuheben, dass das technische Management bei der Durchführung dieser Behandlungsoptionen lediglich eine adjuvante Therapie im Rahmen der chirurgischen Interventionen darstellt. 


\section{Literatur}

${ }^{1}$ Berger A, Hierner R. Neue Entwicklungen bei der Deckung posttraumatischer Weichteildefekte. Orthopäde 1997; 26: 470-480

${ }^{2}$ Bhandari M, Schemtisch EH, Adili A, Lachowski R, Saughnessy SG. High an low pressure pulsatile Lavage of contaminate tibial fractures: An in vitro study of bacterial adhearance and bone damage. J Orthop Trauma 1999; 13(8): 526-533

${ }^{3}$ Breusch SJ, Aldinger PR, Thomsen M, Ewerbeck V, Lukoschek M. Verankerungsprinzipien in der Hüftendoprothetik. Unfallchirurg 2000; 103, 11: 918-931

${ }^{4}$ Buchholz H, Elson RA, Engelbrecht E et al. Management of deep infection of total hip replacement. J Bone Joint Surg Br 1981; 63B: $342-53$

${ }^{5}$ Fleischmann W, Becker U, Bischoff M, Hoekstra $H$. Vacuum sealing: Indication, technique and results. Eur J Orth Surg Taumatol 1995; 5: $37-40$

${ }^{6}$ Geipel U, Herrmann M. The infected implant. Part 1: bacteriology. Orthopäde 2004; 33-12: 1411-1426, 27-28

${ }^{7}$ Perdreau-Remington F, Stefanik D, Peters G, Ludwig C, Rutt J, Wenzel R, Pulverer G. A four-year prospective study on microbia ecology of explanted prosthetic hips in 52 patients with „aseptic“ prosthetic joint loosening. Eur J Clin Microbiol Infect Dis 1996; $15-2: 160-165$

${ }^{8}$ Przybilsky M, Deb R, Ermann D, Germann G. Aktuelle Trends in der Entwicklung von Hautersatzmaterialien. Chirurg 2004; 75: 579-587

${ }^{9}$ Tautenhahn J, Bürger T, Lippert H. Stand der Vakuumversiegelung. Chirurg 2004; 75: $492-497$

${ }^{10}$ Willy C, Sterk J, Gerngro H, Schmidt R. Drainagen in der Weichteilchirurgie. Chirurg 2003: 74: 108-114

${ }^{11}$ Wirbel R, Mutschler W. Postoperative Wunddrainage in der Chirurgie des Bewegungsapparates. Opert Orthop Traumatol 2001; 4: 314-318

${ }^{12}$ Wolter D, Zeifang B, Jürgens C, Neikes M, Wille A. Bedeutung der Keimbesiedelung von Redon-Drainage-Systemen in der Unfallchirurgie. Trauma Berufskrankh 2000; 2: $216-219$

${ }^{13}$ Zalavras CG, Patzakis MJ, Holtom P. Local antibiotic therapy in the treatment of open fractures and osteomyelitis. Clin Orthop Relat Res 2004; 427: 86-93

${ }^{14}$ Ziegler UE, Debus ES, Keller HP, Thiede A. Hautersatzverfahren bei chronischen Wunden. Zentralbl Chir 2001; 126 (Suppl 1): $71-74$
Atesch Ateschrang

Assistenzarzt

Christian Bahrs

Assistenzarzt

Ingo Flesch

Assistenzarzt

Prof. Dr. med. Kuno Weise

Ärztl. Direktor

BG-Unfallklinik

Schnarrenbergstr. 95

D-72076 Tübingen 